\title{
COARSE-GRAINING AND THERMODYNAMICS IN FAR-FROM-EQUILIBRIUM SYSTEMS*
}

\author{
J. Miguel Rubí, A. Pérez-Madrid \\ Departament de Física Fonamental, Facultat de Física, Universitat de Barcelona \\ Martí Franqués 1, 08028 Barcelona, Spain
}

(Received April 11, 2013)

\begin{abstract}
Lying at the core of statistical physics is the need to reduce the number of degrees of freedom in a system. Coarse-graining is a frequently-used procedure to bridge molecular modeling with experiments. In equilibrium systems, this task can be readily performed; however in systems outside equilibrium, a possible lack of equilibration of the eliminated degrees of freedom may lead to incomplete or even misleading descriptions. Here, we present some examples showing how an improper coarse-graining procedure may result in linear approaches to nonlinear processes, miscalculations of activation rates and violations of the fluctuation-dissipation theorem.
\end{abstract}

DOI:10.5506/APhysPolB.44.913

PACS numbers: 05.70.Ln, 05.40.-a, 05.10.Gg

\section{Introduction}

Large-scale systems in equilibrium strictly follow the laws of thermodynamics [1]. Despite the disordered motion of large numbers of molecules, the system can be characterized by a few variables accounting for average properties. Thermodynamics tacitly performs a coarse-graining description of systems that considerably simplifies the characterization of their equilibrium states and the identification of the processes leading from a state to another.

Coarse-graining is also used in nonequilibrium situations when the small parts of the system can be assimilated to small thermodynamic systems in equilibrium. The existence of local equilibrium states makes it possible to analyze the irreversible processes behind the evolution of the systems (aged systems) through the study of the dissipation quantified by the entropy production rate [2].

* Presented at the XXV Marian Smoluchowski Symposium on Statistical Physics, "Fluctuation Relations in Nonequilibrium Regime", Kraków, Poland, September 10-13, 2012. 
Nevertheless, in far-from-equilibrium situations, the coarse-graining description does not always account for the dynamical properties of the system. The degrees of freedom that are eliminated might not yet be equilibrated and, therefore, they would continue to exert an influence in the overall behavior of the system. One could assume that in the coarsened description the system is in equilibrium or local equilibrium when in reality it is not.

In this article, we will present some situations illustrating the fact that by coarsening, a system which is in or close to equilibrium becomes, in fact, far-from-equilibrium. In particular, we will show that by coarsening a diffusion process (which is linear and in local equilibrium) it transforms into an activation process which is nonlinear and, therefore, local equilibrium no longer holds. We will also show that in the process of formation of structures via nucleation or self-assembly, the activation barriers in the reduced description in which the structures are at rest differ from those obtained when the structures undergo Brownian motion. Finally, we will prove that a drastic elimination of some of the degrees of freedom of the system may lead to violation of the fluctuation-dissipation theorem due to the lack of equilibration.

The article is organized as follows. In Section 2, we will show how thermodynamics provides a multi-scale description of the systems linking molecular modeling with experimental conditions. In Section 3, we will show that activated processes result from the elimination of molecular degrees of freedom from a diffusion process. In Section 4, we will show that coarsening modifies the potential barriers and has then implications in the expression of the activation rates. In Section 5, we will demonstrate that coarsening may cause violation of the fluctuation-dissipation theorem. Finally, in Section 6, we will present our main conclusions.

\section{Thermodynamics: a multiscale description of many-particle systems}

Thermodynamics provides a multi-scale description of systems composed by a great number of particles, from large scales to local scales and then to the mesoscale. The way to go from large to local scales is performed in nonequilibrium thermodynamics for systems in local equilibrium [2]. In this section, we will show that a probabilistic interpretation of thermodynamics enables one to extend the applicability of thermodynamic concepts deep into the mesoscopic domain [3].

To this purpose, we should first notice that a reduction of the observational scales of a system usually implies an increase in the number of degrees of freedom which have not yet equilibrated and that, therefore, exert an influence in the overall dynamics of the system [4]. The nonequilibrated degrees 
of freedom could be the velocity of a particle, the orientation of a spin, the size of a macromolecule or any coordinate or order parameter whose values define the state of the system in a phase space. These will be denoted by means of the set of coordinates $\gamma\left(\equiv\left\{\gamma_{i}\right\}\right)$. The characterization of the state of the system is performed through the knowledge of the probability density $P(\gamma, t)$.

To show how thermodynamics applies at the mesoscale, we will start with the statistical expression of the entropy of the system in terms of the probability distribution expressed by the Gibbs entropy postulate [2, 5]

$$
S=S_{\mathrm{eq}}-k_{\mathrm{B}} \int P(\gamma, t) \ln \frac{P(\gamma, t)}{P_{\mathrm{eq}}(\gamma)} d \gamma
$$

Here, $S_{\text {eq }}$ is the entropy when the degrees of freedom $\gamma$ are in equilibrium. The equilibrium probability distribution $P_{\mathrm{eq}}(\gamma)$ can be related to the minimum reversible work required to create that state [9] through the expression

$$
P_{\mathrm{eq}} \sim \exp \left(\frac{-\Delta W(\gamma)}{k_{\mathrm{B}} T}\right)
$$

Here, $k_{\mathrm{B}}$ is Boltzmann's constant and $T$ is the temperature of the heat bath. The minimum work can, in general, be expressed in the form $\Delta W=y \Delta Y$, where $y$ is an intensive parameter and $Y$ its conjugated extensive variable. This general form, which reduces to the different thermodynamic potentials [7], stands for mechanical, electrical, magnetic, surface work, etc. performed on the system [6]. The statistical mechanics definition of the entropy is, therefore, crucial to connect thermodynamics with both the mesoscopic description in terms of the probability distribution $P(\gamma, t)$ and the equilibrium behavior of the system.

The dynamics of the mesoscopic degrees of freedom can be analyzed from the statistical mechanics definition of the entropy. Taking variations in Eq. (1), one obtains

$$
\delta S=-k_{\mathrm{B}} \int \delta P(\gamma, t) \ln \frac{P(\gamma, t)}{P_{\mathrm{eq}}(\gamma)} d \gamma
$$

Conservation of the probability density in $\gamma$-space implies that the probability density obeys the continuity equation

$$
\frac{\partial P}{\partial t}=-\frac{\partial J}{\partial \gamma}
$$

where $J(\gamma, t)$ is a current in the space of mesoscopic coordinates. 
To derive the expression of this current, we take the time derivative in equation (3) and use the continuity equation (4) to eliminate the probability time derivative. After a partial integration, one then arrives at the expression of the mesoscopic entropy production

$$
\sigma=-k_{\mathrm{B}} \int J(\gamma, t) \frac{\partial}{\partial \gamma}\left(\ln \frac{P(\gamma, t)}{P_{\mathrm{eq}}(\gamma)}\right) d \gamma
$$

This quantity is expressed in the form of current-force pairs, the latter being the gradients in the space of mesoscopic variables. We will now assume a linear dependence between current and force and establish the linear relationship

$$
J(\gamma, t)=-k_{\mathrm{B}} L(\gamma, P(\gamma)) \frac{\partial}{\partial \gamma}\left(\ln \frac{P(\gamma, t)}{P_{\mathrm{eq}}(\gamma)}\right),
$$

where $L(\gamma, P(\gamma))$ is an Onsager coefficient [2, 8], which, in general, depends on the probability $P(\gamma)$ interpreted as a state variable in the thermodynamic sense and on the mesoscopic coordinates $\gamma$.

The kinetic equation follows by substituting Eq. (6) into the continuity equation (4)

$$
\frac{\partial P}{\partial t}=\frac{\partial}{\partial \gamma}\left(D P_{\mathrm{eq}} \frac{\partial}{\partial \gamma} \frac{P}{P_{\mathrm{eq}}}\right)
$$

where the diffusion coefficient is defined as $D(\gamma) \equiv k_{\mathrm{B}} L(\gamma, P) / P$. This equation, which in view of Eq. (2) can also be written as

$$
\frac{\partial P}{\partial t}=\frac{\partial}{\partial \gamma}\left(D \frac{\partial P}{\partial \gamma}+\frac{D}{k_{\mathrm{B}} T} \frac{\partial \Delta W}{\partial \gamma} P\right)
$$

is the Fokker-Planck equation for the evolution of the probability density in $\gamma$-space. Under the conditions for which the minimum work is given by the Gibbs free energy $G, \Delta W \equiv \Delta G=\Delta H-T \Delta S$, where $H$ is the enthalpy, this equation transforms into the Fokker-Planck equation for a system in the presence of a free energy barrier

$$
\frac{\partial P}{\partial t}=\frac{\partial}{\partial \gamma}\left(D \frac{\partial P}{\partial \gamma}+\frac{D}{k_{\mathrm{B}} T} \frac{\partial \Delta G}{\partial \gamma} P\right) .
$$

The Fokker-Planck equation (9) contains the work previously defined in the drift term. Therefore, any alteration in the definition of work [6] would invalidate the Fokker-Planck equation and give rise to a completely different dynamics. 


\section{From diffusion to activation}

Nonequilibrium thermodynamics describes irreversible processes in the linear response domain in which the response of the system is linear in the perturbation exerted to remove it from equilibrium. This domain is adequate for many transport processes, even in the presence of large gradients [9, 10], whereas it is not appropriate for activated processes in which the system needs a minimum amount of energy to proceed. Thus, the regime in which they may take place is essentially nonlinear. The difference between transport and activated processes becomes evident when we contrast the form of Fourier, Fick, or Ohm laws, in which the corresponding currents are proportional to the conjugated thermodynamic forces or gradients, with the exponential laws for the rates in activated processes. Activated processes are frequently modeled by a particle crossing a free energy barrier that separates two well-differentiated states located at the minima at each side of the barrier [11, 12].

For activated processes, nonequilibrium thermodynamics describes the process only in terms of the initial and final positions, obtaining a linear behavior of the current in terms of the affinity which only agrees with the law of mass action for small values of the affinity [13]. If we consider the process at shorter time scales, instead of jumping from the initial to the final state, the system progressively transforms by passing through successive molecular configurations. These configurations can be parametrized by a "reaction coordinate" $\gamma$. At these time scales, one may assume that the system undergoes a diffusion process through a potential barrier separating the initial from the final states. These ideas are illustrated in Figs. 1 and 2. Figure 1 corresponds to a cyclical chemical reaction while Fig. 2 represents a cascade of activated processes which corresponds, among other physical situations, to transport at the nanoscale.

In this section, we will show that the activation kinetics is the result of a coarse-graining of the description performed in terms of a diffusion process in the space covered by the reaction coordinate. To this end, we will consider a diffusion process governed by Fick's law

$$
J(\gamma, t)=-\frac{L}{T} \frac{\partial \mu}{\partial \gamma} .
$$

Here, $L$ is an Onsager coefficient and $\mu$ the chemical potential defined in $\gamma$-space

$$
\mu=k_{\mathrm{B}} T \ln P+\Phi,
$$

where $\Phi$ is an external potential. The diffusion current can also be expressed in the form

$$
J(\gamma, t)=-D \frac{\partial \tilde{P}}{\partial \gamma}
$$


with the fugacity $\tilde{P}=P \exp \left[\Phi /\left(k_{\mathrm{B}} T\right)\right]$, where $\exp \left[\Phi /\left(k_{\mathrm{B}} T\right)\right]$ is the activity coefficient, and the diffusion coefficient is given by

$$
D=D_{0} \exp \left(-\frac{\Phi}{k_{\mathrm{B}} T}\right)=\frac{k_{\mathrm{B}} L}{P} \exp \left(-\frac{\Phi}{k_{\mathrm{B}} T}\right)
$$

where $D_{0}$ is the diffusion coefficient in the absence of potential.

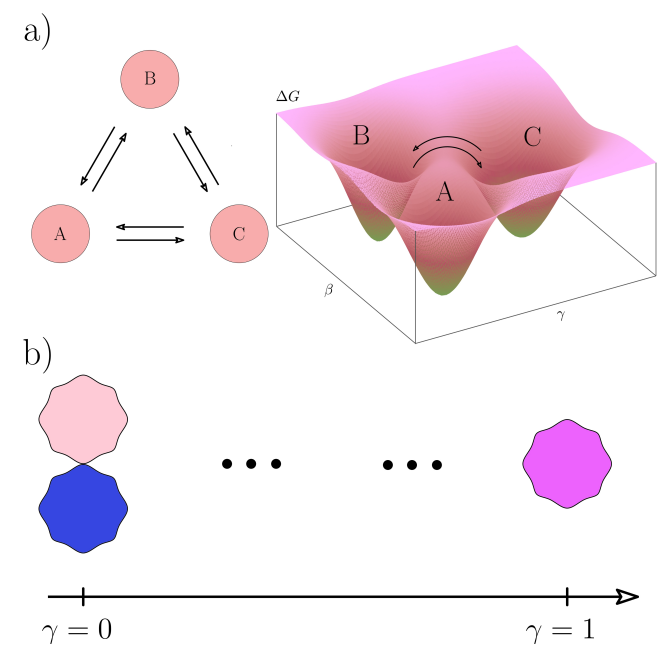

Fig. 1. (a) Transformations of the molecules of a biochemical cycle viewed as a diffusion process in a two-dimensional free-energy landscape. The configurations are parametrized by means of two coordinates $\gamma$ and $\beta$. The molecular structure of a substance transforms progressively until it reaches its final conformation. (b) In the coarse-grained level, the initial and final structures are described by the reaction coordinate $\gamma$ when $\beta$ is maintained constant.

a)

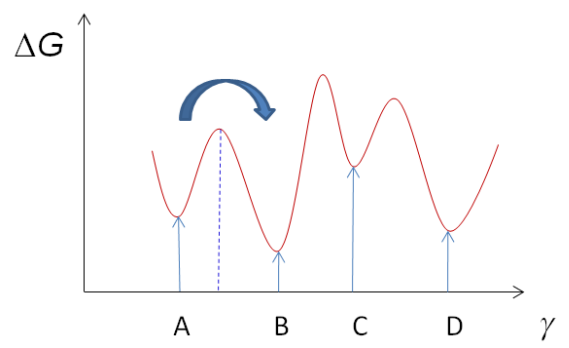

b)

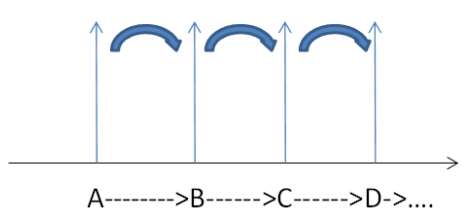

Fig. 2. Variation of the Gibbs free energy landscape as a function of the reaction coordinate $\gamma$. (a) Full description. (b) Coarse-grained description. 
The contraction of the description is performed by integrating Eq. (12) from 1 to 2. This leads to the activation current $J(t)$ as a combination of two Arrhenius factors for the forward and backward processes

$$
\begin{aligned}
J(t) & =-\left\langle\exp \left(\frac{\Phi}{k_{\mathrm{B}} T}\right)\right\rangle^{-1} \frac{D_{0}}{l}\left(\tilde{P}_{2}-\tilde{P}_{1}\right) \\
& =-\left\langle\exp \left(\frac{\Phi}{k_{\mathrm{B}} T}\right)\right\rangle^{-1} \frac{D_{0}}{l}\left[\exp \left(\frac{\mu_{2}}{k_{\mathrm{B}} T}\right)-\exp \left(\frac{\mu_{1}}{k_{\mathrm{B}} T}\right)\right],
\end{aligned}
$$

where $l$ is the distance between states 1 and 2 .

The obtained result shows how to remove the limitations of linearity of nonequilibrium thermodynamics in the description of activated processes. When thermodynamics is applied at short time scales in which the transformation from the initial to the final state is sufficiently slow, one can use local equilibrium in the space of the reaction coordinate to describe the underlying diffusion process. A contraction of the description then leads to the nonlinear activation kinetics.

\section{Far-from-equilibrium kinetic processes}

To show how the coarse-graining affects the activation kinetics, we will consider the case of formation of clusters or structures of different sizes from a metastable phase via nucleation $[14,15]$ or self-assembly processes. We will assume that the variables characterizing the structures are the number of building blocks $n$, the translational velocity $\vec{u}$, and the rotational velocity $\vec{\omega}$. The description of the system will be carried out in terms of the distribution function $P(\underline{\Gamma}, t)$, with $\underline{\Gamma}=\underline{\Gamma}(n, \vec{u}, \vec{\omega})$, whose evolution is governed by the continuity equation

$$
\frac{\partial P(\underline{\Gamma}, t)}{\partial t}=-\frac{\partial}{\partial \underline{\Gamma}} \cdot \underline{J}(\underline{\Gamma}, t),
$$

where $\underline{J}=\left(J_{n}, J_{u}, J_{\omega}\right)$ is a current defined in $\underline{\Gamma}$-space.

As we have shown in the previous section, at sufficiently short time scales the kinetic of formation of structures can be assimilated to a diffusion process in a potential in $\underline{\Gamma}$-space. The energy barrier is in this case given by

$$
C(\underline{\Gamma})=\Phi(n)+\frac{1}{2} m u^{2}+\frac{1}{2} I \omega^{2},
$$

where $\Phi(n)$ represents the energy of formation of a $n$-structure at rest of mass $m$ and moment of inertia $I$. Following the steps indicated in Section 2, we arrive at the Fokker-Planck equation

$$
\frac{\partial P}{\partial t}=\frac{\partial}{\partial \underline{\Gamma}} \cdot\left(\underline{D} \cdot \frac{\partial P}{\partial \underline{\Gamma}}+\frac{\underline{D}}{k_{\mathrm{B}} T} \cdot \frac{\partial C}{\partial \underline{\Gamma}} P\right),
$$


where $\underline{D}=k_{\mathrm{B}} \underline{L} / P$ is the matrix of diffusion coefficients. This equation describes the kinetics of the process in terms of the number of building blocks of the structure and of its velocities.

It is a common feature that the velocities equilibrate in a Brownian time scale much shorter than that for $n$. Consequently, inertial effects are negligible and one can proceed with a simplified description of the process in the diffusion regime in $n$-space. To obtain the corresponding kinetic equation, we will perform an adiabatic elimination of the velocities. The resulting Fokker-Planck equation is given by

$$
\frac{\partial P(n, t)}{\partial t}=\frac{\partial}{\partial n}\left[D_{\mathrm{eff}} \frac{\partial P}{\partial n}+\frac{D_{\mathrm{eff}}}{k_{\mathrm{B}} T}\left(\frac{d \Phi}{d n}+4 k_{\mathrm{B}} T \frac{1}{n}\right) P\right] .
$$

Here, the effective diffusion coefficient $D_{\text {eff }}$ can be identified with the forward rate of the process [15]. This equation corresponds to diffusion in $n$-space over the effective activation barrier

$$
\Delta G(n)=\Phi(n)+4 k_{\mathrm{B}} T \ln n,
$$

where the logarithmic term results from the Brownian motion of the structure in the metastable phase. This term contributes to the increase of the potential barrier and, consequently, to the reduction of the rate. The increase is due to the fact that every time that a structure captures or releases a molecule not only the size distribution is altered but also the velocity distribution. The modification of cluster size distribution entails a reorganization of velocity distribution through diffusing currents. These currents involve an additional dissipation which has an energetic cost. Consequently, the growing of the cluster requires more effective energy because part of that is expended in the reorganization of the velocity distribution.

\section{Coarse-graining induced violation of the fluctuation-dissipation theorem}

The lack of equilibration of the degrees of freedom that are eliminated when coarse-graining the description may lead to violation of the fluctuationdissipation theorem [16], a result which is valid strictly under equilibrium or local equilibrium conditions. To show this fact, we will consider the case of diffusion in a bistable potential defined in $\gamma$-space.

In Section 2, we have shown that in such a situation the probability distribution satisfies the Fokker-Planck equation

$$
\frac{\partial P(\gamma, t)}{\partial t}=\frac{\partial}{\partial \gamma} D\left(\frac{\partial P(\gamma, t)}{\partial \gamma}+\frac{P(\gamma, t)}{k_{\mathrm{B}} T} \frac{\partial \Phi(\gamma)}{\partial \gamma}\right) .
$$


This equation has the form of a diffusion equation in the presence of a force and can be formulated when the random variable $\gamma$ varies in a small amount over a small time interval, when the noise is Gaussian. Under these conditions, the random current of the corresponding Langevin equation satisfies the fluctuation-dissipation theorem

$$
\left\langle J^{\mathrm{r}}(\gamma, t) J^{\mathrm{r}}\left(\gamma^{\prime}, t^{\prime}\right)\right\rangle=2 D\langle P(\gamma, t)\rangle \delta\left(\gamma-\gamma^{\prime}\right) \delta\left(t-t^{\prime}\right) .
$$

We will now define the integrated probability density

$$
N(\gamma, t)=\int_{-\infty}^{\gamma} P\left(\gamma^{\prime}, t\right) d \gamma^{\prime}
$$

This quantity satisfies the equation

$$
\frac{\partial}{\partial t} N(\gamma, t)=-J^{\mathrm{s}}(\gamma, t)-J^{\mathrm{r}}(\gamma, t),
$$

where $J^{\mathrm{s}}(\gamma, t)$ is the systematic part of the current. To eliminate the reaction coordinate, we will use the integral operator $\mathscr{P}$ defined through its action on a unspecified function in $\gamma$-space

$$
\mathscr{P} \Psi(\gamma, t)=\frac{1}{\int_{\gamma_{1}}^{\gamma_{2}} d \gamma \exp \left[\Phi /\left(k_{\mathrm{B}} T\right)\right]} \int_{\gamma_{1}}^{\gamma_{2}} d \gamma \exp \left(\frac{\Phi}{k_{\mathrm{B}} T}\right) \Psi(\gamma, t) .
$$

This operator acts on the currents in the form: $J^{\alpha}(t)=\mathscr{P} J^{\alpha}(\gamma, t)$.

Applying it to Eq. (23), we obtain

$$
\frac{d n_{1}}{d t}=-\frac{d n_{2}}{d t}=-J^{\mathrm{s}}(t)-J^{\mathrm{r}}(t),
$$

where

$$
J^{\mathrm{s}}(t)=k_{\rightarrow} n_{1}-k_{\leftarrow} n_{2},
$$

and $n_{1}$ and $n_{2}$ are "populations" of each one of the minima. Here, the forward and backward rates are given by

$$
k_{\rightarrow, \leftarrow}=\frac{D}{2 \pi k_{\mathrm{B}} T}\left(\Phi^{\prime \prime}\left(\gamma_{1,2}\right)\left|\Phi^{\prime \prime}\left(\gamma_{0}\right)\right|\right)^{1 / 2} \exp \left\{\frac{\Phi\left(\gamma_{1,2}\right)-\Phi\left(\gamma_{0}\right)}{k_{\mathrm{B}} T}\right\} .
$$

Applying the operator $\mathscr{P}$ to both sides of the fluctuation-dissipation theorem (21), we obtain the correlation of the random part in the reduced description

$$
\left\langle J^{\mathrm{r}}(t) J^{\mathrm{r}}\left(t^{\prime}\right)\right\rangle=\left(k_{\rightarrow}\left\langle n_{1}\right\rangle+k_{\leftarrow}\left\langle n_{2}\right\rangle\right) \delta\left(t-t^{\prime}\right) .
$$


This expression does not correspond to the fluctuation-dissipation theorem. Only in equilibrium, when $k_{\rightarrow} n_{1}^{\mathrm{eq}}=k_{\leftarrow} n_{2}^{\mathrm{eq}}$, this expression reduces to the fluctuation-dissipation theorem [16]

$$
\left\langle J^{\mathrm{r}}(t) J^{\mathrm{r}}\left(t^{\prime}\right)\right\rangle_{\mathrm{eq}}=2 k_{\rightarrow} n_{1}^{\mathrm{eq}} \delta\left(t-t^{\prime}\right) .
$$

The theorem, which holds in $\gamma$-space, fails due to the coarsening of the description. When the dynamics is analyzed in terms of diffusion in the reaction coordinate, the system progressively passes from one state to the other, which supports the assumption of local equilibrium in $\gamma$-space and consequently makes the formulation of a mesoscopic nonequilibrium thermodynamics possible. However, when we describe the system over longer time intervals, we are only capturing the activated process which is not near equilibrium and accordingly, the fluctuation-dissipation theorem does not hold.

\section{Conclusions}

Thermodynamics furnishes a multi-scale description of many-particle systems: a global description adequate for equilibrium systems [1], a local description for systems in local equilibrium [2] and a mesoscopic description for systems in local equilibrium at the mesoscale [4]. To provide a bridge across these scales, one has to use coarse-graining which eliminates fine interaction details. It might seem that the reduction of variables can be systematically applied, whereas in reality this is not so. Some of the eliminated degrees of freedom could be coupled to those remaining thereby having implications in the dynamics.

In this article, we have shown some situations in which coarse-graining has consequences in the thermodynamic description. In the study of activated processes, nonequilibrium thermodynamics performs a coarsened description reducing the states to two, the initial and the final states. The possible outcome of this choice is the linearity between rates and affinities that describe the process when the system is close to equilibrium. If, on the contrary, one considers all the molecular configurations along the path of the reaction coordinate, one obtains the nonlinear behavior expected. We have demonstrated as well that in an activated process the correct way to coarsen the description is to retain all the degrees of freedom before performing an adiabatic elimination of the faster variables. Finally, we have shown that in the coarsened description, the fluctuation-dissipation theorem could not be fulfilled. 
The Authors would like to thank J.M.G. Vilar from CSIC-UPV/EHU, David Reguera, and Ignacio Pagonabarraga from the Universitat de Barcelona and Fernando Bresme from the Imperial College, London. This work has been supported by the Icrea Academia Program and by the MINECO of the Spanish government through the Grant No. FIS2008-01299.

\section{REFERENCES}

[1] H.B. Callen, Thermodynamics and an Introduction to Thermostatistics, John Wiley and Sons, Inc., New York 1985.

[2] S.R. de Groot, P. Mazur, Non-Equilibrium Thermodynamics, Dover, New York 1984.

[3] D. Reguera, J.M. Rubi, J.M.G. Vilar, J. Phys. Chem. 109, 21502 (2005).

[4] J.M.G. Vilar, J.M. Rubi, Proc. Nat. Acad. Sci. 98, 11081 (2001).

[5] N.G. van Kampen, Stochastic Processes in Physics and Chemistry, North-Holland, Amsterdam 1987.

[6] J.M.G. Vilar, J.M. Rubi, Phys. Rev. Lett. 100, 020601 (2008).

[7] L.D. Landau, E.M. Lifshitz, Statistical Physics, Pergamon Press, New York 1989.

[8] J.W. Dufty, J.M. Rubi, Phys. Rev. A36, 222 (1987).

[9] A. Barreiro et al., Science 320, 775 (2008).

[10] F. Römer et al., Phys. Rev. Lett. 108, 105901 (2012).

[11] H. Kramers, Physica 7, 284 (1940).

[12] P. Hänggi, P. Talkner, M. Borkevec, Rev. Mod. Phys. 62, 251 (1990).

[13] I. Pagonabarraga, A. Pérez-Madrid, J.M. Rubi, Physica A 237, 205 (1997).

[14] D. Reguera, J.M. Rubi, J. Chem. Phys. 109, 5987 (1998).

[15] D. Reguera, J.M. Rubi, J. Chem. Phys. 115, 7100 (2001).

[16] A. Pérez-Madrid, D. Reguera, J.M. Rubi, Physica A 329, 357 (2003). 